\title{
Long-term bicycle riding ameliorates the depression of the patients undergoing hemodialysis by affecting the levels of interleukin- 6 and interleukin- 18
}

\author{
Chunhui Zhao \\ Hui Ma \\ Lei Yang \\ Yong Xiao
}

Blood Purification Center, The First Affiliated Hospital of Dalian Medical University, Dalian, People's Republic of China
Correspondence: Yong Xiao Blood Purification Center, The First Affiliated Hospital of Dalian Medical University, No 222 Zhongshan Road, Xigang District, Dalian, Liaoning Province, People's Republic of China

Tel/fax +864II 83635963

Email xyong2016@163.com
This article was published in the following Dove Press journal:

Neuropsychiatric Disease and Treatment

28 December 2016

Number of times this article has been viewed

Purpose: Hemodialysis patients with depression have a higher risk of death and hospitalization. Although there is pharmacological management for the depression of hemodialysis patients, the adverse effect of the drug limits the use. The nonpharmacological way, bicycle riding, may be an effective way for the therapy of the depression in hemodialysis patients. However, the underlying mechanism of this relationship is still not fully explained, while interleukin-6 (IL-6) and interleukin-18 (IL-18) are associated with depression and exercise. Thus, the effects of bicycle riding on the levels of the interleukin were explored.

Participants and methods: One hundred and eighty-nine patients with chronic hemodialysis were selected and randomly assigned to three groups of medicine (MG, received 20-mg escitalopram daily), medicine and aerobic exercise (MAG, received 20-mg escitalopram daily and bicycle riding six times weekly), and only aerobic exercise (AG, received 20-mg placebo daily and bicycle riding six times weekly). The whole experiment lasted for 18 weeks. The quality of life (36-Item Short Form Health Survey) and depression severity according to criteria in the Diagnostic and Statistical Manual of Mental Disorders, fourth edition [DSM-IV] were measured before and at the end of this study. The serum levels of IL- 6 and IL-18 were measured by an enzyme-linked immunosorbent assay kit.

Results: The quality of life was improved and depression severity was reduced significantly in the MAG and AG groups when compared with the MG group $(P<0.05)$. Serum levels of IL-6 and IL-18 were the highest in the MG group, moderate in the MAG group and the lowest in AG group. On the other hand, the serum levels of IL-6 and IL-18 were closely associated with depression scores $(P<0.05)$.

Conclusion: Aerobic exercise improves the quality of life and ameliorates the depression severity of the patients undergoing hemodialysis by affecting the levels of IL-6 and IL-18. Bicycle riding is a potential way for the depression therapy of the patients with chronic hemodialysis.

Keywords: aerobic exercise, depression, hemodialysis patients

\section{Introduction}

Depression is one of the main symptoms in patients receiving hemodialysis. ${ }^{1,2}$ This disease is characterized by pervasive feelings of sadness, ${ }^{3,4}$ irritation, ${ }^{5,6}$ low self-esteem ${ }^{7}$ and poor sleep, ${ }^{8,9}$ which directly affect the quality of life of the patients; especially, anxiety and depression feelings are mental problems. More seriously, depression is closely associated with suicidal events. ${ }^{10,11}$ There are some diagnostic features according to the international Beck Depression Inventory-II (BDI-II) with 21 questions about 
how the patients feel in the last week: 1) do not feel sad; 2) feel sad; 3 ) feel sad all the time and cannot snap out of it and 4) feel so sad or unhappy that he or she cannot stand it. ${ }^{12}$ The severity of depression was indicated with scores from 0 to 3 according to their answers. ${ }^{13}$

Although the standard for depression diagnosis has been set for decades, the pathophysiological process is complex and more molecular mechanisms for causing depression are needed to be confirmed. Antidepressant medication is still the main way for depression therapy. For example, the selective serotonin re-uptake inhibitor (SSRI) antidepressant escitalopram (Lexapro) has been widely used for the treatment of major depression. The 17-Item Hamilton Rating Scale for Depression (HAMD-17) analysis indicates that generic escitalopram is effective and safe in the therapy of patients with moderate to severe depression. ${ }^{14}$ Although the data suggest the effectiveness and safety of generic escitalopram for the therapy of depression patients, the side effects are serious, including drowsiness, ${ }^{15}$ diarrhea, ${ }^{16}$ nausea, ${ }^{15}$ vomiting, ${ }^{15}$ ejaculatory dysfunctions, ${ }^{17}$ insomnia ${ }^{16}$ and headache. ${ }^{16}$ All the unwanted side effects limit the usage of the drug.

Therefore, it is highly necessary to explore nonpharmaceutical therapy. Aerobic exercise has been demonstrated to be beneficial to the therapy of depression patients and has no side effects. ${ }^{18,19}$ The method has also been approved in the patients suffering from chronic hemodialysis. ${ }^{20,21}$ However, the molecular mechanism for the role of aerobic exercise in the therapy of depression remains widely unclear. Some evidence shows that inflammation is associated with depression disorder. ${ }^{22}$ Most studies also indicated that inflammatory cytokines and immune-derived products play an important role in the major depressive disorder. ${ }^{23}$ There are many cytokines that are related to inflammatory processes. Interleukin-6 (IL-6) and interleukin-18 (IL-18) are associated with exercise. Furthermore, IL-6 has emerged as a biomarker for detecting depression as its high level in depressive patients was confirmed by meta-analyses. ${ }^{24}$ IL-18, as a proinflammatory cytokine, plays a critical role in the T-cell-helper type 1 response and is a member of cytokines produced in the brain. ${ }^{25}$ High levels of IL-6 and IL-18 are found to be independently associated with depression, and the results are stable even after 1 year. ${ }^{26}$ Thus, we aimed to assess the effect of aerobic exercise on depression severity and patients' quality of life during chronic hemodialysis by investigating the changes in IL- 6 and IL-18.

\section{Participants and methods}

\section{Participants}

Before the experiment, all the protocols were approved by the ethical committees of The First Affiliated Hospital of
Dalian Medical University (reference number: HK2016-48). Written informed consent was obtained from all the participants. All patients received hemodialysis with any etiology of renal failure and depression symptoms in the First Affiliated Hospital of Dalian Medical University. There were three phases in the study. In phase I, a qualitative study was performed among community members and health experts. In this way, all participants understood the terms that were used to express their symptoms, which were adapted from the Chinese version of BDI-II. In phase II, the Chinese version of BDI-II was used among all the participants to assess the psychometric characteristics of BDI. In phase III, the Chinese version of BDI-II was used to assess the discriminant validity of BDI-II. A total of 326 patients were enrolled in this randomized clinical trial. After the administration of BDI-II, all participants were questioned to assess each item on relevancy, lucidity of item phrasing, and correctness for the context. Written notes were analyzed independently by different experts to identify recurring themes.

\section{Measurement}

The BDI-II was modified according to the severity of depression from non-symptom (0) to severe symptom (3) within 14 days. ${ }^{27}$ The scores of depression were classified as weak (0-13), mild (14-19), moderate (20-28), and severe (29-63) according to the criteria in Diagnostic and Statistical Manual of Mental Disorders, fourth edition (DSM-IV). ${ }^{28}$

\section{Inclusion criteria}

The inclusion criteria were as follows: 1) the hemodialysis had been performed for at least 3 months or at least three times within 1 week and 2) the participant had the ability to ride a bicycle.

\section{Exclusion criteria}

Patients with the following disorders were excluded: 1) opportunistic infections; 2) medical therapy for other diseases during the last 3 months; 3) systolic blood pressure $>160 \mathrm{mmHg}$ and/or diastolic blood pressure $>110 \mathrm{mmHg}$ before and after hemodialysis and/or at hours 2 and 3 during hemodialysis; 4) symptoms for interrupting the exercises, such as chest pain, dyspnea, body temperature $>38^{\circ} \mathrm{C}$ and cardiac arrhythmias; 5) signs of neurological vertigo and/or imbalance and 6) nonadherence to the exercise program and instability in hemodynamic parameters after exercises.

\section{Grouping}

All of the participants (361 patients) participated in our study, and 189 patients were selected, after considering the 
inclusion and exclusion criteria, and randomly assigned to the medicine group (MG, 63 patients received 20-mg escitalopram daily), medicine and aerobic exercise group (MAG, 63 patients received 20-mg escitalopram daily and bicycle riding six times weekly) and aerobic exercise group (AG, 63 patients received 20-mg placebo daily and bicycle riding six times weekly). The participants in the MAG and AG groups rode Forever-brand bicycles (Shanghai Forever Bicycle Company, Shanghai, the People's Republic of China) at least six times weekly for 18 weeks. Each time, the exercise lasted for at least 1 hour after hemodialysis. To avoid cardiovascular dysfunction, the first 5 minutes were spent for warm-up by performing some easy activities. The length of the whole road was $10 \mathrm{~km}$ without an obvious increase at elevation, and the bicycle riding speed was hoped to be kept at $20 \mathrm{~km} / \mathrm{h}$. The participants were asked to avoid other powerful physical activities. The participants were required to sit for $<5$ minutes one time and no more than two times if they were too tired to ride the bicycle during the exercise. The whole bicycle riding time was 1 hour. After 1-hour bicycleriding exercise, cooldown was conducted to lower heartbeat at the final 5 minutes. The standard for Borg-scale exercise was used to evaluate exercise intensity. ${ }^{29}$ Quality of life was evaluated by using 36-Item Short Form Health Survey (SF-36) questionnaire ${ }^{30}$ before and after 18-week study.

\section{Analysis of side effects at end points}

Escitalopram treatment can cause some side effects. Thus, the side effects (headache, nausea, diarrhea, dry mouth, ${ }^{31}$ sleepiness, ${ }^{32}$ insomnia, constipation ${ }^{33}$ and yawning ${ }^{34}$ ) were measured. All patients had no such side effects before this study.

\section{ELISA analysis of serum levels of IL-6 and IL- I 8}

A total of $5 \mathrm{~mL}$ of venous blood was obtained from each participant. Venous blood was centrifuged at $3,000 \times g$ for 10 minutes, and the serum was collected. A microtiter plate was coated with serum sample in each well. The concentrations of serum levels of IL- 6 and IL-18 were measured by using Human IL-6 ELISA Kit (Cat No ab46042, reliability level was from 1 to $100 \mathrm{pg} / \mathrm{mL}$ ) and Human IL-18 ELISA Kit (Cat No ab46032, reliability level was from 100 to 2,000 pg/mL) from Abcam (Cambridge, MA, USA).

\section{Statistical analysis}

Student's $t$-test and chi-square statistics test were used for comparing the data between two groups. One-way analysis of variance (ANOVA) was performed among the three groups. All data are presented as mean \pm SD and analyzed by SPSS 20 (IBM Corporation, Armonk, NY, USA). Statistically significant differences were considered if $P<0.05$ with two-sided $90 \%$ confidence intervals.

\section{Results \\ Baseline characteristics}

Of the participants, 120 patients $(63 \%)$ were males and 69 patients $(37 \%)$ were females. Mean age of the participants was $56.9 \pm 17.5$ years (Table 1 ). The scores of depression were similar before the experiment, and there was no significant difference among these groups in terms of life styles, comorbidities, SF-36 questions and depression severity $(P>0.05)$.

\section{Evaluation of quality of life}

Assessment of quality of life indicated that mean scores were similar among the three groups before the experiment $(P>0.05)$. After 18-week aerobic exercise, average scores were higher in the AG group than in the MAG group and were further higher in the $\mathrm{MG}$ group $(P<0.05$; Table 2$)$. The results suggest that aerobic exercise improved the quality of life of patients undergoing hemodialysis, while escitalopram may affect the quality of life of patients, with some side effects in the MAG group compared with the AG group.

\section{Depression severity evaluation}

After 18-week therapy, seven, eight and four patients with severe depression withdrew from the experiment in the $\mathrm{MG}$, AG and MAG groups, respectively. Therefore, 56, 55 and 59 patients completed the study finally in the three groups, respectively (Figure 1). The differences in the mean values among the three groups suggested that long-term bicycle riding improved depression symptoms of the patients undergoing hemodialysis. Before the study, there was no statistically significant difference in non-symptom and severe depression among the three groups $(P<0.05$; Table 1$)$. Comparatively, there were statistically significant differences in non-symptom and severe depression among the three groups after the 18 -week trial $(P<0.05)$. The results suggest that bicycle riding ameliorated the depression severity of the patients undergoing hemodialysis, while escitalopram showed less effectivity in the MAG group, with some side effects compared with the AG group.

\section{Side effects of escitalopram}

As shown in Table 3, the side effects of escitalopram in the patients included headache, nausea, diarrhea, dry mouth, sleepiness, insomnia, constipation and yawning. The risk 
Table I Demographic characteristics among the three groups

\begin{tabular}{|c|c|c|c|c|}
\hline Variable & MG (\%) & AG (\%) & MAG (\%) & $P$-value \\
\hline Gender (male), n (\%) & $40(63.5)$ & $4 I(65.1)$ & $39(61.9)$ & 0.934 \\
\hline Age (years), mean $(90 \% \mathrm{Cl})$ & $54.1(42.3-68.7)$ & $53.6(44.5-66.3)$ & $52.9(43.9-65.8)$ & 0.456 \\
\hline Smoker/non-smoker, n/n & $30 / 33$ & $31 / 32$ & $29 / 34$ & 0.938 \\
\hline Drinker/non-drinker, $\mathrm{n} / \mathrm{n}$ & $29 / 34$ & $30 / 33$ & $31 / 32$ & 0.938 \\
\hline Education levels (>9 years), n (\%) & $20(31.7)$ & $22(34.9)$ & $19(30.2)$ & 0.844 \\
\hline Spouse, n (\%) & $56(88.9)$ & $59(93.7)$ & $57(90.5)$ & 0.636 \\
\hline Career (working overtime), n (\%) & $30(47.6)$ & $34(54.0)$ & $33(52.4)$ & 0.759 \\
\hline Time on hemodialysis (months), mean $(90 \% \mathrm{Cl})$ & $24.7(15.6-36.1)$ & $25.3(14.9-34.2)$ & $24.9(13.8-35.7)$ & 0.627 \\
\hline Dialysis efficacy, mean $(90 \% \mathrm{Cl})$ & $0.67(0.6 \mathrm{I}-0.73)$ & $0.64(0.49-0.78)$ & $0.75(0.66-0.84)$ & 0.893 \\
\hline Duration of hemodialysis (hours), mean $(90 \% \mathrm{Cl})$ & $3.12(2.5 \mathrm{I}-3.64)$ & $3.07(2.64-3.4 I)$ & $2.93(2.45-3.53)$ & 0.782 \\
\hline \multicolumn{5}{|l|}{ Comorbidities, $n$ (\%) } \\
\hline DM & $14(22.2)$ & $12(19)$ & $13(20.6)$ & 0.908 \\
\hline HTN & $15(23.8)$ & $17(27.0)$ & $17(27.0)$ & 0.896 \\
\hline Others (glomerular disease, polycystic kidney, urine stone) & $10(15.9)$ & II (I7.5) & $12(19.0)$ & 0.896 \\
\hline DM and HTN & $13(20.6)$ & $15(23.8)$ & $14(22.2)$ & 0.850 \\
\hline Unknown & $7(I I . I)$ & $5(7.9)$ & $6(9.5)$ & 0.832 \\
\hline \multicolumn{5}{|l|}{ SF-36, mean $(90 \% \mathrm{Cl})$} \\
\hline Vitality & $36.2(24.8-46.5)$ & $35.4(25.6-44.9)$ & $37.5(24.1-48.0)$ & 0.312 \\
\hline Physical functioning & $50.2(34.1-63.4)$ & $48.5(32.1-56.0)$ & $49.2(36.7-52.4)$ & 0.625 \\
\hline Bodily pain & $44.2(31.2-57.8)$ & $46.7(33.9-60.4)$ & $45.4(32.6-58.3)$ & 0.426 \\
\hline General health & $43.9(29.8-57.6)$ & $42.1(27.4-58.0)$ & $44.2(30.5-58.6)$ & 0.552 \\
\hline Physical role functioning & $54.3(40.2-70.4)$ & $55.2(42.7-68.3)$ & $54.9(43.6-71.1)$ & 0.325 \\
\hline Emotional role functioning & $44.2(29.5-54.5)$ & $43.8(28.6-55.3)$ & $42.8(30.1-56.0)$ & 0.569 \\
\hline Social role functioning & $58.4(47.5-69.2)$ & $57.3(48.6-72.5)$ & $55.6(49.7-68.5)$ & 0.176 \\
\hline Mental health & $46.3(38.5-55.4)$ & $47.1(37.9-57.2)$ & $46.8(36.1-56.3)$ & 0.512 \\
\hline \multicolumn{5}{|l|}{ Depression severity } \\
\hline Non-symptom (0-13) & 8 & 11 & 13 & 0.489 \\
\hline Mild (14-19) & 5 & 5 & 9 & 0.392 \\
\hline Moderate (20-28) & 7 & 4 & 8 & 0.467 \\
\hline Severe $(29-63)$ & 42 & 43 & 33 & 0.128 \\
\hline
\end{tabular}

Notes: A total of 189 patients were selected after considering inclusion and exclusion criteria and randomly assigned to medicine group (MG, only received escitalopram $20 \mathrm{mg} /$ day; 63 patients), medicine and aerobic exercise group (MAG; 63 patients), and aerobic exercise group (AG, placebo was used; 63 patients). Chi-square test and Student's $t$-test were performed among MG, AG and MAG groups. Statistically significant differences were considered if $P$ was $<0.05$.

Abbreviations: $\mathrm{Cl}$, confidence interval; DM, diabetes mellitus; HTN, hypertension; SF-36, 36-Item Short Form Health Survey.

Table 2 Clinical characteristics among the three groups after 18-week therapy

\begin{tabular}{|c|c|c|c|c|}
\hline Variable & MG (\%) & AG (\%) & MAG (\%) & $P$-value \\
\hline \multicolumn{5}{|l|}{ SF-36, mean $(90 \% \mathrm{Cl})$} \\
\hline Vitality & $50.4(38.1-51.3)$ & $71.6(57.2-86.2)$ & $57.2(47.9-69.1)$ & 0.018 \\
\hline Physical functioning & $64.2(51.8-78.4)$ & $76.9(64.4-89.8)$ & $68.8(62.1-85.3)$ & 0.024 \\
\hline Bodily pain & $52.7(4 I .2-63.5)$ & $70.3(56.8-84.1)$ & $64.6(52.1-76.8)$ & 0.028 \\
\hline General health & $52.2(40.8-60.4)$ & $76.9(65.3-88.3)$ & $65.1(51.6-78.0)$ & 0.010 \\
\hline Physical role functioning & $54.4(39.8-69.1)$ & $73.0(58.6-89.4)$ & $63.2(49.5-76.2)$ & 0.021 \\
\hline Emotional role functioning & $54.1(42.3-58.7)$ & $68.5(55.4-81.3)$ & $61.8(46.5-76.2)$ & 0.033 \\
\hline Social role functioning & $53.7(38.9-69.1)$ & $76.4(60.3-92.4)$ & $66.4(54.7-79.5)$ & 0.018 \\
\hline Mental health & $55.3(41.6-65.8)$ & $74.6(65.2-86.8)$ & $60.9(47.5-73.1)$ & 0.005 \\
\hline \multicolumn{5}{|l|}{ Depression severity } \\
\hline Non-symptom (0-13) & 16 & 29 & 21 & 0.027 \\
\hline Mild (14-19) & 8 & 16 & 12 & 0.159 \\
\hline Moderate (20-28) & 9 & 10 & 11 & 0.929 \\
\hline Severe $(29-63)$ & 23 & 0 & 15 & 0.001 \\
\hline
\end{tabular}

Notes: A total of 189 patients were selected after considering the inclusion and exclusion criteria and randomly assigned to the medicine group (MG, only received escitalopram 20 mg/day; 63 patients), medicine and aerobic exercise group (MAG; 63 patients), and aerobic exercise group (AG, placebo was used; 63 patients). Chi-square test and Student's $t$-test were performed among the MG, AG and MAG groups. Statistically significant differences were considered if $P$ was $<0.05$.

Abbreviations: SF-36, 36-Item Short Form Health Survey; Cl, confidence interval. 


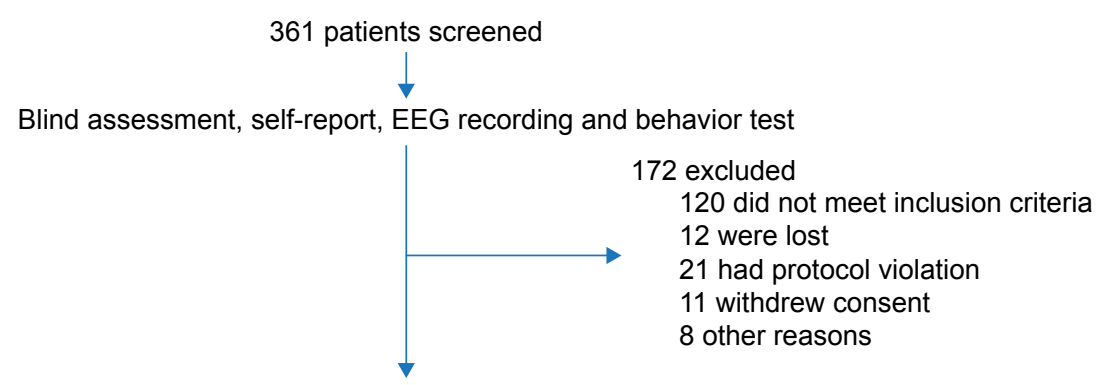

189 underwent randomization

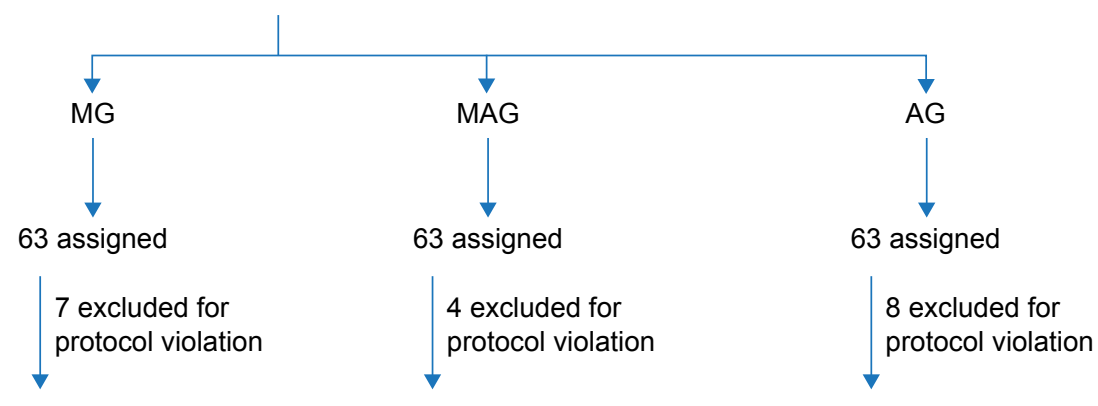

56 completed the study

59 completed the study

55 completed the study

Figure I The flowchart of the present study.

Note: All the experiments lasted for 18 weeks.

Abbreviations: EEG, electroencephalography; MG, medicine group; MAG, medicine and aerobic exercise group; AG, aerobic exercise group.

rates of side effects were higher in the MG group than in the MAG group, which were further higher than in the AG group. There were statistically significant differences in diarrhea, sleepiness and constipation between the MG and AG groups $(P<0.05$; Table 3$)$. Therefore, the aerobic exercise bicycle riding reduced the side effects of escitalopram.

\section{Changes in serum concentrations of IL-6 and IL- 18}

The serum levels of IL-6 (Figure 2A) and IL-18 (Figure 2B) were lower in the $\mathrm{AG}$ group than in the MAG group, which were further lower than in the MG group $(P<0.05)$. IL-6 and IL-8 are important biomarkers for the severity of depression. ${ }^{26}$ Aerobic exercise therapy greatly reduced the levels of IL-6 and IL-18. There were significant differences in the serum levels of IL- 6 and IL-18 among the three groups after the 18-week trial $(P<0.05$; Figure 2$)$.

\section{Serum concentrations of IL-6 were closely associated with the severity of depression}

The serum concentrations of IL-6 were closely associated with the severity of depression $(P<0.05$; Figure 3$)$. In the MG group, the scores of depression severity were positively related with serum levels of IL-6 before (Figure 3A) and after (Figure 3B) the therapy. Similarly, the scores of depression severity were positively related with serum levels of IL-6 before (Figure 3C) and after (Figure 3D) the therapy in

Table 3 The comparison for the side effects of escitalopram

\begin{tabular}{|c|c|c|c|c|c|c|}
\hline Side effects & $M G(n=56)$ & AG $(n=55)$ & MAG $(n=59)$ & $P I$ value & P2 value & $P 3$ value \\
\hline Headache, $n(\%)$ & $9(16.1)$ & $4(7.3)$ & 7 (II.9) & 0.149 & 0.515 & 0.407 \\
\hline Nausea, n (\%) & II (19.6) & $5(9.1)$ & $8(13.6)$ & 0.114 & 0.380 & 0.453 \\
\hline Diarrhea, n (\%) & $13(23.2)$ & $3(5.5)$ & $10(16.9)$ & 0.008 & 0.401 & 0.054 \\
\hline Dry mouth, n (\%) & $6(10.7)$ & $7(12.7)$ & $5(8.5)$ & 0.672 & 0.683 & 0.460 \\
\hline Sleepiness, n (\%) & $8(14.2)$ & $\mathrm{I}(\mathrm{I} .8)$ & $4(6.8)$ & 0.040 & 0.188 & 0.404 \\
\hline Insomnia, n (\%) & $9(16.1)$ & $2(3.6)$ & $6(10.2)$ & 0.028 & 0.348 & 0.318 \\
\hline Constipation, n (\%) & $10(17.9)$ & $3(5.5)$ & $8(13.6)$ & 0.042 & 0.526 & 0.143 \\
\hline Yawning, n (\%) & $9(16.1)$ & $4(7.3)$ & 7 (II.9) & 0.149 & 0.515 & 0.407 \\
\hline
\end{tabular}

Notes: A total of 189 patients were selected after considering the inclusion and exclusion criteria and randomly assigned to the medicine group (MG, only received escitalopram 20 mg/day; 63 patients), medicine and aerobic exercise group (MAG; 63 patients), and aerobic exercise group (AG, placebo was used; 63 patients). Chi-square test was performed between the two groups. PI presents the comparison between MG and AG groups, P2 presents the comparison between MG and MAG groups and $P 3$ presents the comparison between AG and MAG groups. Statistically significant differences were considered if $P$ was $<0.05$. 
A

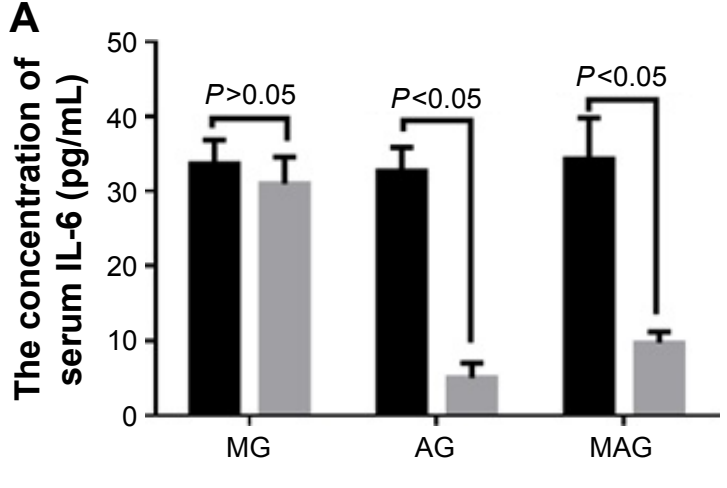

B

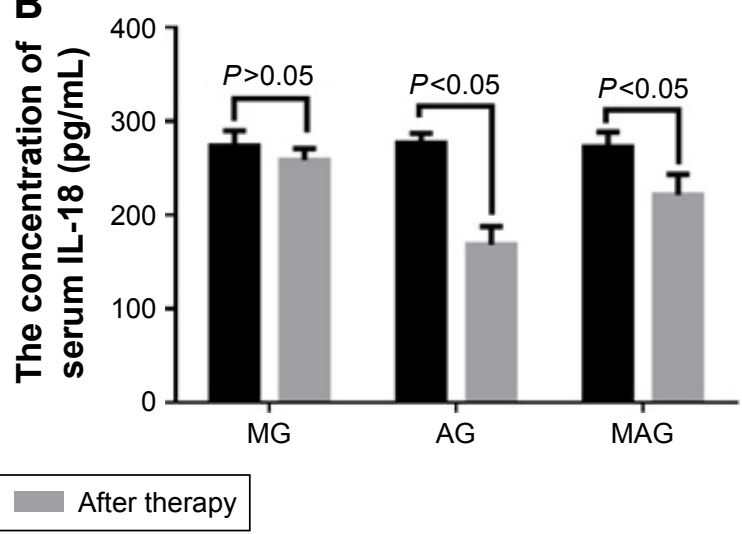

Figure 2 The effects of serum IL-6 and IL-18 on the severity of depression.

Notes: A total of 189 patients were selected after considering the inclusion and exclusion criteria and randomly assigned to the medicine group (MG, only received escitalopram 20 mg/day; 63 patients), medicine and aerobic exercise group (MAG; 63 patients), and aerobic exercise group (AG, placebo was used; 63 patients). (A) The effects of serum IL- 6 on the severity of depression. (B) The effects of serum IL-I 8 on the severity of depression. $* P<0.05$ vs before therapy.

Abbreviations: IL-6, interleukin-6; IL-18, interleukin-18.

the AG group. The scores of depression severity were also positively related to serum levels of IL-6 before (Figure 3E) and after (Figure 3F) the therapy in the MAG group. The levels of IL-6 were the lowest in the AG group, suggesting that the aerobic exercise ameliorates depression severity by affecting serum levels of IL-6.

\section{Serum concentrations of IL- 18 were closely associated with the severity of depression}

The serum concentrations of IL-18 were closely associated with the severity of depression $(P<0.05$; Figure 4$)$. In the MG group, the scores of depression severity were positively related with serum levels of IL-18 before (Figure 3A) and after (Figure 3B) the therapy. Similarly, the scores of depression severity were positively related to serum levels of IL-18 before (Figure 3C) and after (Figure 3D) therapy in the AG group. The scores of depression severity were also positively related to serum levels of IL-18 before (Figure 3E) and after (Figure 3F) the therapy in the MAG group. The levels of IL-6 were the lowest in the AG group, suggesting that the aerobic exercise ameliorates depression severity by affecting serum levels of IL-18.

\section{Discussion}

We aimed to set up a non-pharmacological interaction for ameliorating depression severity of the patients with chronic hemodialysis. Depression is a common symptom in hemodialysis patients, and 126 patients participated in the experiment after considering the inclusion and exclusion criteria. The present findings showed that the quality of life
(SF-36) was significantly improved after long-term bicycle riding training (Table 2). On the other hand, the scores of $D S M-I V$ were more significantly reduced in the AG group than in the MAG group, which were further lower than in the MG group $(P<0.01$; Table 2$)$. Thus, bicycle riding is an excellent way to improve the quality of life and reduce the severity of depression (Table 2) and can be used for patients suffering from depression because of chronic hemodialysis. Theoretically, with the prolongation of the study, the quality of life may be further improved.

Because of adverse effects of antidepression medicine on patients' life, it is very important to explore a perfect way to alleviate adverse side effects and improve the quality of life. The present findings showed that long-term bicycle riding can prevent the side effects caused by escitalopram. Thus, bicycle riding may provide a potential way for reducing the side effects caused by the medicine for depression therapy. Future studies with more patients will elucidate whether the aerobic exercise has more confirming effects on these adverse side effects.

Although bicycle riding is beneficial to the depression therapy for the patients undergoing hemodialysis, the exact molecular mechanism is unclear. To explore the molecular mechanism for the role of bicycle riding in the therapy of depression for the patients undergoing hemodialysis, the changes in important biomarkers such as IL-6 and IL-18 in depression patients were investigated here. The results showed that long-term bicycle riding reduced serum levels of IL-6 and IL-18. Meanwhile, the scores of depression severity were positively related with serum levels of IL-6 (Figure 3) and IL-18 (Figure 4) before and after the therapy. The levels 
A

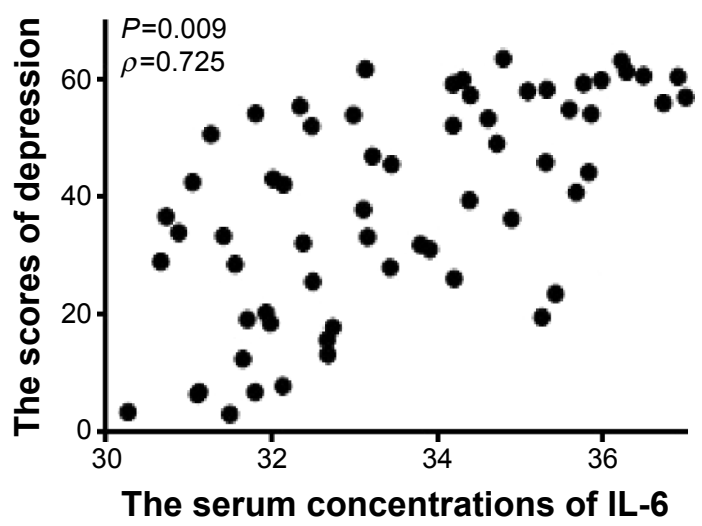

C

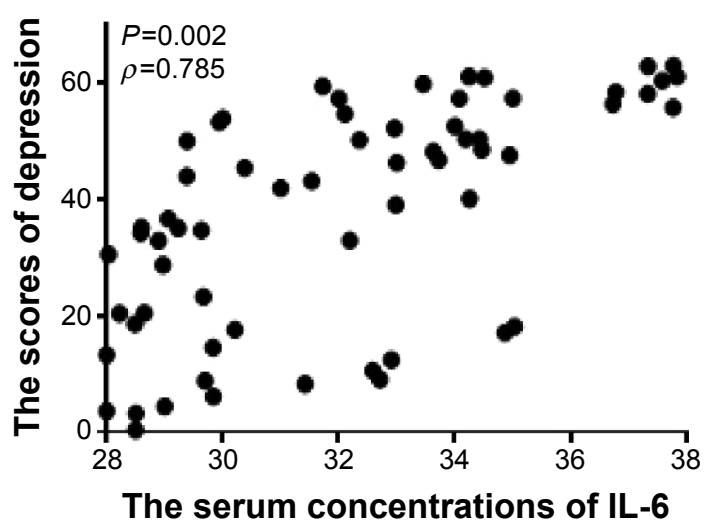

E

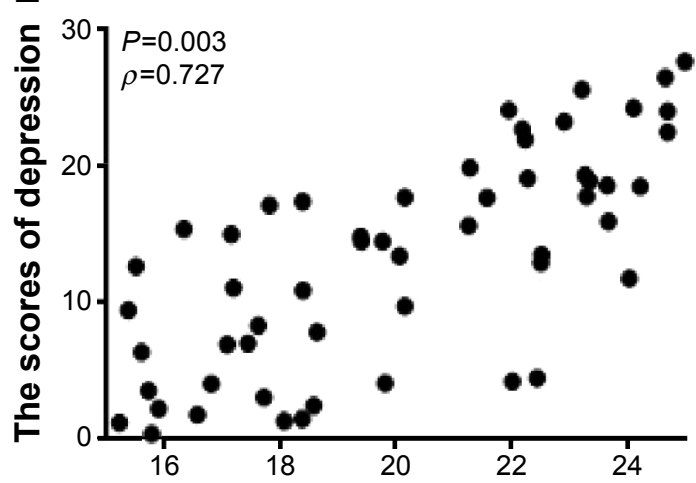

The serum concentrations of IL- 6
B

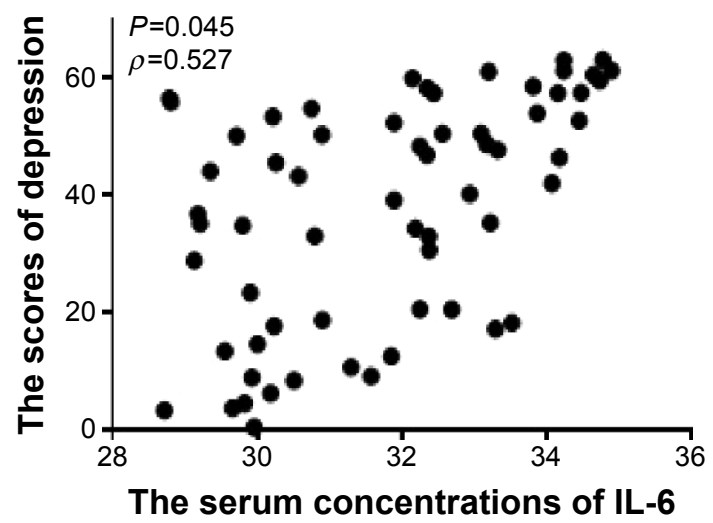

D

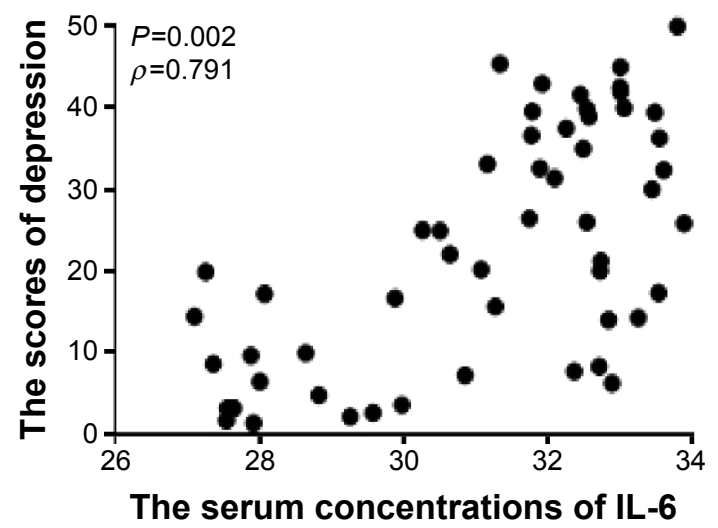

$\mathbf{F}$

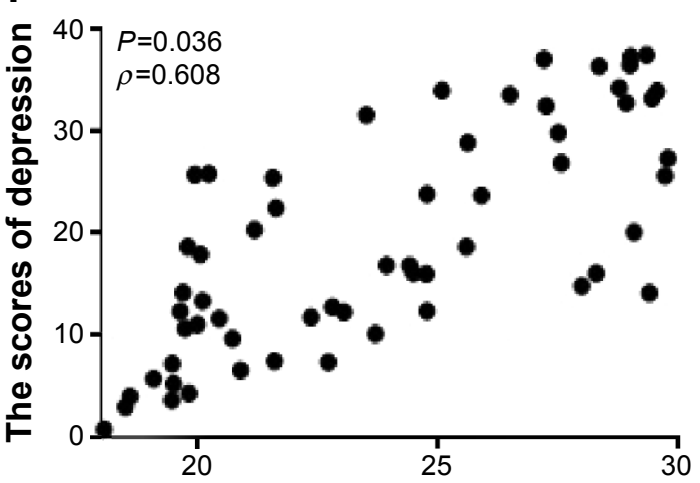

The serum concentrations of IL-6

Figure 3 The association between the scores of depression and serum levels of IL-6.

Notes: A total of 189 patients were selected after considering the inclusion and exclusion criteria and randomly assigned to the medicine group (MG, only received escitalopram 20 mg/day; 63 patients), medicine and aerobic exercise group (MAG; 63 patients), and aerobic exercise group (AG, placebo was used; 63 patients). (A) The association between the scores of depression and serum levels of IL-6 in the MG group before therapy. (B) The association between the scores of depression and serum levels of IL-6 in the MG group after therapy. (C) The association between the scores of depression and serum levels of IL-6 in the AG group before therapy. (D) The association between the scores of depression and serum levels of IL-6 in the AG group after therapy. (E) The association between the scores of depression and serum levels of IL-6 in the MAG group before therapy. (F) The association between the scores of depression and serum levels of IL- 6 in the MAG group after therapy.

Abbreviation: IL-6, interleukin-6.

of IL-6 and IL-18 were the lowest in the AG group, suggesting that aerobic exercise ameliorates depression severity by affecting serum levels of IL-6 and IL-18.

Other mechanisms may also exist but are not considered here. For instance, bicycle riding increases muscle blood flow and an open capillary surface area in working muscles, which will improve a greater flux of urea and remove toxins from the tissue by sweating. Hemodialysis patients have reduced physical function and a higher risk of increased arterial stiffness, which is caused by hypertension, 
A

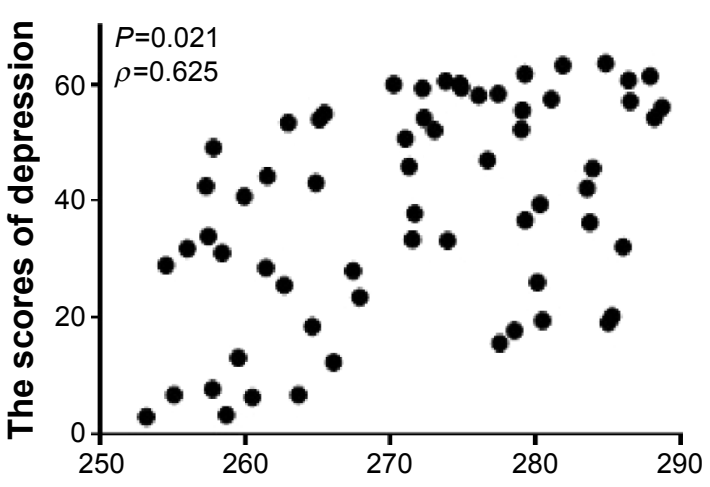

The serum concentrations of IL-18

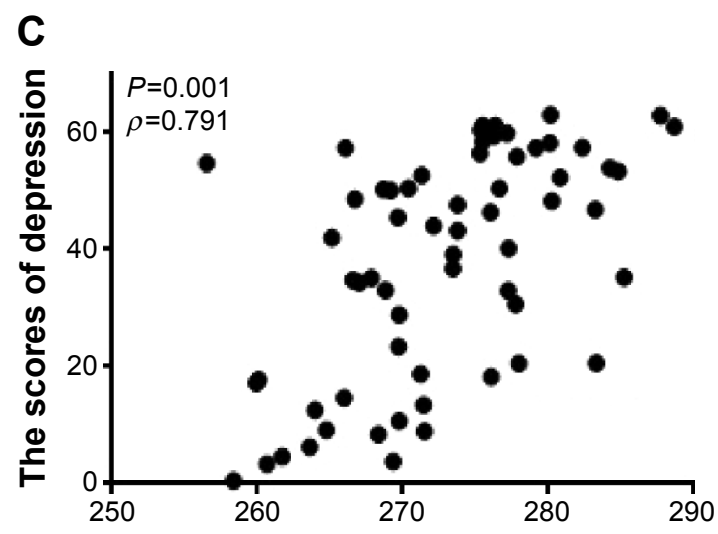

The serum concentrations of IL-18

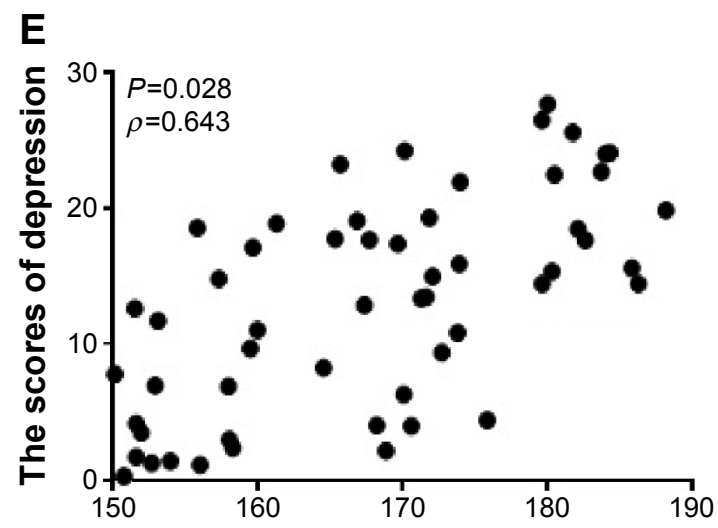

The serum concentrations of IL-18

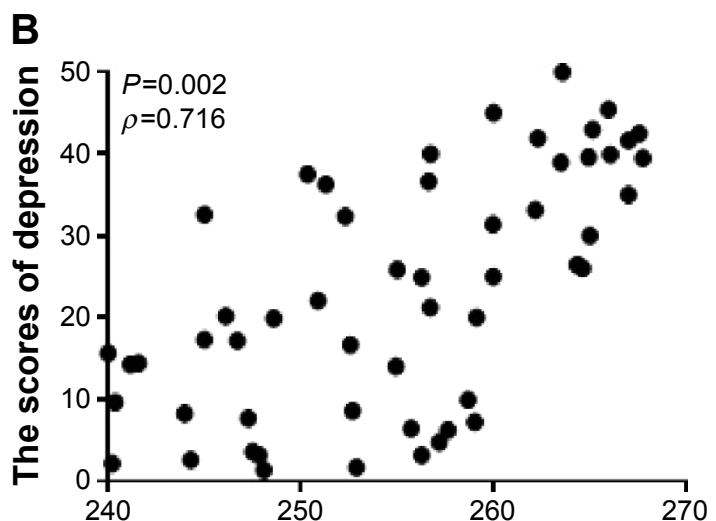

The serum concentrations of IL-18

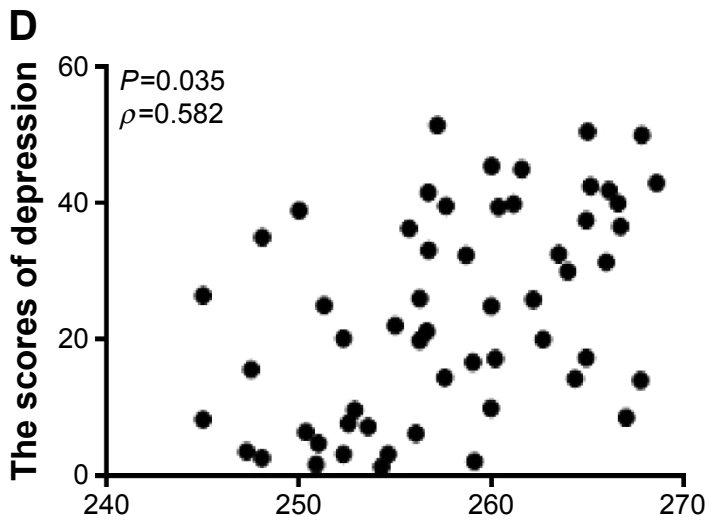

The serum concentrations of IL-18

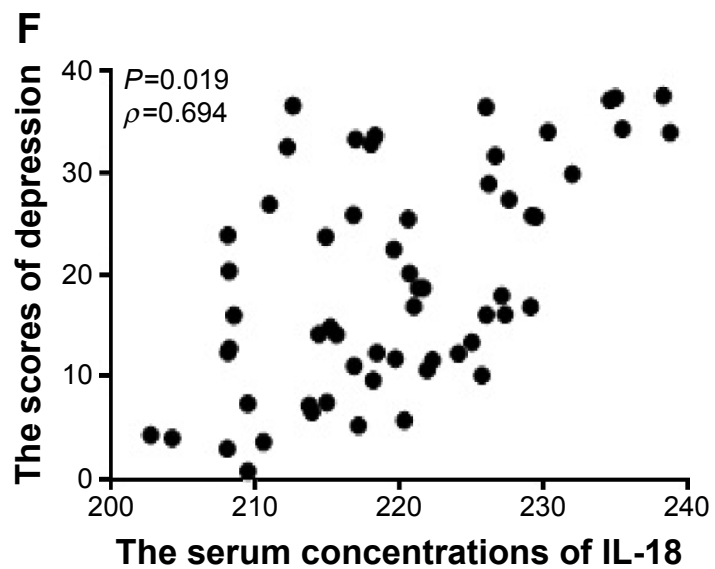

Figure 4 The association between the scores of depression and serum levels of IL-I8.

Notes: A total of 189 patients were selected after considering the inclusion and exclusion criteria and randomly assigned to the medicine group (MG, only received escitalopram 20 mg/day; 63 patients), medicine and aerobic exercise group (MAG; 63 patients), and aerobic exercise group (AG, placebo was used; 63 patients). (A) The association between the scores of depression and serum levels of IL- 18 in the MG group before therapy. (B) The association between the scores of depression and serum levels of IL- 18 in the MG group after therapy. (C) The association between the scores of depression and serum levels of IL-I8 in the AG group before therapy. (D) The association between the scores of depression and serum levels of IL- 18 in the AG group after therapy. (E) The association between the scores of depression and serum levels of IL-I8 in the MAG group before therapy. (F) The association between the scores of depression and serum levels of IL-I8 in the MAG group after therapy.

Abbreviation: IL-18, interleukin- 18.

metabolic disturbances, vascular calcification and so on. Considering the inadequacy of hemodialysis is one main cause of disability and mortality of hemodialysis patients; therefore, aerobic exercise reduces vascular risk profile in hemodialysis patients.
There were some limitations to the present study. 1) The study was performed at one hemodialysis center in the city of Dalian. Therefore, a limited number of hemodialysis patients were recruited and the sample size was small for making a more confirming conclusion. A larger and multicenter study 
will be performed in the future. 2) The intensity of exercise was controlled by a cycle ergometer. The heart rate of patients was used to measure the exercise intensity. 3) We could not find a good control group although the aerobic exercise was performed well in an experiment group. 4) The work was not performed in a healthy population. According to the previous study, different exercises show different effects on the level of IL-6 in healthy patients. ${ }^{35}$ Another report indicates that the exercise will result in a small release of IL- 8 from the muscles, but this cannot cause the increase in the plasma level of IL- 8 in healthy people. ${ }^{36}$

\section{Conclusion and recommendations}

Long-term bicycle riding improves the quality of life (SF-36) and reduces the severity of depression in patients suffering from chronic hemodialysis. The aerobic exercise reduces the levels of IL-6 and IL-18. Meanwhile, the serum levels of IL-6 and IL-18 were related to the scores of depression $(P<0.05)$. Thus, long-term bicycle riding may ameliorate the depression of the patients undergoing hemodialysis by affecting the levels of IL-6 and IL-18. To obtain more confirmed results, much work is needed to confirm the molecular mechanism for the critical role of aerobic exercise in improving the quality of life of hemodialysis patients.

\section{Acknowledgment}

We are very grateful to all anonymous reviewers for their critical and strategic comments, which have significantly improved the quality of the present article.

\section{Disclosure}

The authors report no conflicts of interest in this work.

\section{References}

1. King-Wing Ma T, Kam-Tao Li P. Depression in dialysis patients. Nephrology (Carlton). 2016;21(8):639-646.

2. Dziubek W, Kowalska J, Kusztal M, et al. The level of anxiety and depression in dialysis patients undertaking regular physical exercise training - a preliminary study. Kidney Blood Press Res. 2016;41(1):86-98.

3. Bartczak M, Bokus B. Semantic distances in depression: relations between ME and PAST, FUTURE, JOY, SADNESS, HAPPINESS. $J$ Psycholinguist Res. Epub 2016 Jun 23.

4. Drageset J, Eide GE, Hauge S. Symptoms of depression, sadness and sense of coherence (coping) among cognitively intact older people with cancer living in nursing homes-a mixed-methods study. PeerJ. 2016; 4:e2096.

5. Sibelli A, Chalder T, Everitt H, Workman P, Windgassen S, MossMorris R. A systematic review with meta-analysis of the role of anxiety and depression in irritable bowel syndrome onset. Psychol Med. 2016: $1-16$.

6. Jizhong S, Qiaomin W, Chao W, Yanqing L. Corticotrophin-releasing factor and toll-like receptor gene expression is associated with low-grade inflammation in irritable bowel syndrome patients with depression. Gastroenterol Res Pract. 2016;2016:7394924.
7. Krug S, Wittchen HU, Lieb R, Beesdo-Baum K, Knappe S. Family functioning mediates the association between parental depression and low self-esteem in adolescents. J Affect Disord. 2016;203:184-189.

8. Woods HC, Scott H. \#Sleepyteens: social media use in adolescence is associated with poor sleep quality, anxiety, depression and low selfesteem. J Adolesc. 2016;51:41-49.

9. Tham EK, Tan J, Chong YS, et al. Associations between poor subjective prenatal sleep quality and postnatal depression and anxiety symptoms. $J$ Affect Disord. 2016;202:91-94.

10. Brent D, Melhem N, Ferrell R, et al. Association of FKBP5 polymorphisms with suicidal events in the Treatment of Resistant Depression in Adolescents (TORDIA) study. Am J Psychiatry. 2010;167(2):190-197.

11. Horesh N, Nachshoni T, Wolmer L, Toren P. A comparison of life events in suicidal and nonsuicidal adolescents and young adults with major depression and borderline personality disorder. Compr Psychiatry. 2009; 50(6):496-502.

12. Beck AT, Ward CH, Mendelson M, Mock J, Erbaugh J. An inventory for measuring depression. Arch Gen Psychiatry. 1961;4:561-571.

13. Beck AT, Steer RA, Carbin MG. Psychometric properties of the Beck Depression Inventory: twenty-five years of evaluation. Clin Psychol Rev. 1988;8(1):77-100.

14. Yu Y, Li H, Wang B, et al. Efficacy and safety of generic escitalopram versus Lexapro in the treatment of major depression: a multicenter double-blinded randomized controlled trial. Shanghai Arch Psychiatry. 2013;25(2):107-115.

15. Hayes BD, Klein-Schwartz W, Clark RF, Muller AA, Miloradovich JE. Comparison of toxicity of acute overdoses with citalopram and escitalopram. J Emerg Med. 2010;39(1):44-48.

16. Findling RL, Robb A, Bose A. Escitalopram in the treatment of adolescent depression: a randomized, double-blind, placebo-controlled extension trial. J Child Adolesc Psychopharmacol. 2013;23(7):468-480.

17. Koyuncu H, Serefoglu EC, Yencilek E, Atalay H, Akbas NB, Sarica K. Escitalopram treatment for premature ejaculation has a negative effect on semen parameters. Int J Impot Res. 2011;23(6):257-261.

18. Sadeghi K, Ahmadi SM, Ahmadi SM, et al. A comparative study of the efficacy of cognitive group therapy and aerobic exercise in the treatment of depression among the students. Glob J Health Sci. 2016; 8(10):54171.

19. El-Rafie MM, Khafagy GM, Gamal MG. Effect of aerobic exercise during pregnancy on antenatal depression. Int $J$ Womens Health. 2016; 8:53-57.

20. Shahgholian N, KarimiFard O, Shahidi S. Effects of aerobic exercise on blood glucose in continuous ambulatory peritoneal dialysis patients. Iran J Nurs Midwifery Res. 2015;20(2):165-170.

21. Mohseni R, Emami Zeydi A, Ilali E, Adib-Hajbaghery M, Makhlough A. The effect of intradialytic aerobic exercise on dialysis efficacy in hemodialysis patients: a randomized controlled trial. Oman Med J. 2013;28(5): 345-349.

22. Haroon E, Miller AH. Inflammation effects on brain glutamate in depression: mechanistic considerations and treatment implications. Curr Top Behav Neurosci. Epub 2016 Nov 10.

23. Kudinova AY, Deak T, Hueston CM, et al. Cross-species evidence for the role of interleukin-33 in depression risk. $J$ Abnorm Psychol. 2016;125(4):482-494.

24. Kovacs D, Eszlari N, Petschner P, et al. Interleukin-6 promoter polymorphism interacts with pain and life stress influencing depression phenotypes. J Neural Transm (Vienna). 2016;123(5):541-548.

25. Merendino RA, Di Rosa AE, Di Pasquale G, et al. Interleukin-18 and CD30 serum levels in patients with moderate-severe depression. Mediators Inflamm. 2002;11(4):265-267.

26. Kang HJ, Bae KY, Kim SW, et al. Effects of interleukin-6, -18, and statin use, evaluated at acute stroke, on post-stroke depression during 1-year follow-up. Psychoneuroendocrinology. 2016;72:156-160.

27. Steer RA, Clark DA, Beck AT, Ranieri WF. Common and specific dimensions of self-reported anxiety and depression: the BDI-II versus the BDI-IA. Behav Res Ther. 1999;37(2):183-190.

28. Zinbarg RE, Barlow DH, Liebowitz M, et al. The DSM-IV field trial for mixed anxiety-depression. Am J Psychiatry. 1994;151(8):1153-1162. 
29. Aamot IL, Forbord SH, Karlsen T, Stoylen A. Does rating of perceived exertion result in target exercise intensity during interval training in cardiac rehabilitation? A study of the Borg scale versus a heart rate monitor. J Sci Med Sport. 2014;17(5):541-545.

30. Adoga AA, Kokong DD, Nimkur TL, Okwori ET. The impact of tinnitus on adult Nigerians: health related Quality of Life assessment of sufferers using the Hospital Anxiety and Depression Scale (HADS) and the RAND-36 item health survey 1.0 questionnaire. Int Tinnitus $J$. 2015;19(2):26-32.

31. Bose A, Li D, Gandhi C. Escitalopram in the acute treatment of depression patients aged 60 years or older. Am J Geriatr Psychiatry. 2008; 16(1):14-20.

32. Doerr JP, Spiegelhalder K, Petzold F, et al. Impact of escitalopram on nocturnal sleep, day-time sleepiness and performance compared to amitriptyline: a randomized, double-blind, placebo-controlled study in healthy male subjects. Pharmacopsychiatry. 2010;43(5):166-173.
33. Wade A, Gembert K, Florea I. A comparative study of the efficacy of acute and continuation treatment with escitalopram versus duloxetine in patients with major depressive disorder. Curr Med Res Opin. 2007; 23(7):1605-1614.

34. Roncero C, Mezzatesta-Gava M, Grau-Lopez L, Daigre C. Yawning as a dose-dependent side effect of treatment with escitalopram. Neurologia. 2013;28(9):589-590.

35. Philippe M, Krusmann PJ, Mersa L, et al. Acute effects of concentric and eccentric exercise on glucose metabolism and interleukin-6 concentration in healthy males. Biol Sport. 2016;33(2):153-158.

36. Akerstrom T, Steensberg A, Keller P, Keller C, Penkowa M, Pedersen BK. Exercise induces interleukin-8 expression in human skeletal muscle. J Physiol. 2005;563(pt 2):507-516.

\section{Publish your work in this journal}

Neuropsychiatric Disease and Treatment is an international, peerreviewed journal of clinical therapeutics and pharmacology focusing on concise rapid reporting of clinical or pre-clinical studies on a range of neuropsychiatric and neurological disorders. This journal is indexed on PubMed Central, the 'PsycINFO' database and CAS, and is the official journal of The International Neuropsychiatric Association (INA). The manuscript management system is completely online and includes a very quick and fair peer-review system, which is all easy to use. Visit http://www.dovepress.com/testimonials.php to read real quotes from published authors.

Submit your manuscript here: http://www.dovepress.com/neuropsychiatric-disease-and-treatment-journal 